\title{
Secure Dynamic SSE via Access Indistinguishable Storage
}

\author{
Tianhao Wang ${ }^{*}$ \\ Department of Computer Science \\ Purdue University \\ West Lafayette, USA \\ tianhaowang@purdue.edu
}

\author{
Yunlei Zhao ${ }^{\dagger}$ \\ Key Laboratory of Data Science of Shanghai, \\ School of Computer Science and Engineering \\ Fudan University, Shanghai, China \\ State Key Laboratory of Cryptology \\ Beijing, China \\ ylzhao@fudan.edu.cn
}

\begin{abstract}
Cloud storage services such as Dropbox [1] and Google Drive [2] are becoming more and more popular. On the one hand, they provide users with mobility, scalability, and convenience. However, privacy issues arise when the storage becomes not fully controlled by users. Although modern encryption schemes are effective at protecting content of data, there are two drawbacks of the encryption-before-outsourcing approach: First, one kind of sensitive information, Access Pattern of the data is left unprotected. Moreover, encryption usually makes the data difficult to use.

In this paper, we propose AIS (Access Indistinguishable Storage), the first client-side system that can partially conceal access pattern of the cloud storage in constant time. Besides data content, AIS can conceal information about the number of initial files, and length of each initial file. When it comes to the access phase after initiation, AIS can effectively conceal the behavior (read or write) and target file of the current access. Moreover, the existence and length of each file will remain confidential as long as there is no access after initiation.

One application of AIS is SSE (Searchable Symmetric Encryption), which makes the encrypted data searchable. Based on AIS, we propose SBA (SSE Built on AIS). To the best of our knowledge, SBA is safer than any other SSE systems of the same complexity, and SBA is the first to conceal whether current keyword was queried before, the first to conceal whether current operation is an addition or deletion, and the first to support direct modification of files.
\end{abstract}

\section{Keywords}

Searchable Encryption; Cloud Storage; Access Pattern

\footnotetext{
${ }^{*}$ The first author's work is supported in part by the United States National Science Foundation under Grant No. 1116991.

${ }^{\dagger}$ Contact author. This work is supported by NSFC (Grant Nos. 61472084, 61272012, U1536205).

Permission to make digital or hard copies of all or part of this work for personal or classroom use is granted without fee provided that copies are not made or distributed for profit or commercial advantage and that copies bear this notice and the full citation on the first page. Copyrights for components of this work owned by others than the author(s) must be honored. Abstracting with credit is permitted. To copy otherwise, or republish, to post on servers or to redistribute to lists, requires prior specific permission and/or a fee. Request permissions from permissions@acm.org.
}

ASIA CCS '16, May 30-June 03, 2016, Xi'an, China

(C) 2016 Copyright held by the owner/author(s). Publication rights licensed to ACM. ISBN 978-1-4503-4233-9/16/05 . . \$15.00

DOI: http://dx.doi.org/10.1145/2897845.2897884

\section{INTRODUCTION}

Nowadays, more and more users including individuals, organizations, and companies choose to outsource their data to cloud storage, since it is economic, scalable, and usually available. However, since the cloud storage services are provided by profit-driven companies, users won't trust the cloud if their data is sensitive or valuable. A good practice is to encrypt the data before outsourcing, but such protection is not enough, because the layout of the cloud storage, together with the cloud system calls, which are clear to the cloud owner, could leak information such as the exact number of files, the size of each file, and the Access Pattern.

Access Pattern $(A P)$ is a set of tuples $(t, i d, o p)$, where $t$ is a timestamp, $i d$ is the identification of the data item accessed by the user, and $o p$ is the operation from read, write, create or delete. Each tuple of $A P$ means: at time $t$, the user ' $o p$ ' data item $i d$.

Consequence of access pattern leakage is terrifying. For example, Islam et al. [15] demonstrate statistical attacks that leverage access patterns to successfully infer about $80 \%$ of the search queries made to an encrypted email repository. Cash et al. [6] also systematically studied the security model and attacks, and first proposed active attacks that can recover plaintext.

Access pattern may not be concrete when evaluating its importance, therefore, we extract three kinds of sensitive information from access patterns and give examples to show the consequences of leaking such information. Before that, we distinguish between a 'virtual' access and an 'actual' access.

A cloud access, also called an 'actual' access, is a download or upload of encrypted data located somewhere in the cloud. Counterpart of a cloud access is an access, also called a 'virtual' access. A virtual access is a read, write, create or delete of a plain data item from the user's perspective. Each virtual access corresponds to one or several cloud accesses.

The three kinds of information are linkability, predictability, and frequency of accesses. Informally, two cloud accesses are linked if they correspond to the same virtual access, e.g., reading the same file. Cloud accesses are said to be predictable when the attacker can tell the virtual access, e.g., reading a file, without seeing any consequent behaviors or the victim. And frequencies are the statistics of virtual accesses, e.g., how often does the victim read a specific file.

Since each access consists of two parts: the object being accessed, identified by $i d$, and the operation of the access, $o p$, six examples are given as thought experiments to show 


\begin{tabular}{|l||c|c|}
\hline & $i d$ & $o p$ \\
\hline Linkability & Patient & Government \\
\hline Predictability & Enterprise & Military \\
\hline Frequency & Meta File & Stock Exchange \\
\hline
\end{tabular}

Table 1: Examples

the importance of these three characteristics of either part. The examples are categorized in table 1.

Patient: Personal Health Records (PHR) enables a doctor to manage his patients conveniently. Outsourcing PHRs to the cloud makes it readily accessible in case of emergency. If the adversary can link some access of the record of a patient, and the access that establishes the record of that patient. The patient's sensitive information that he may have some disease is leaked to the adversary.

Government: Government files are of crucial importance, and they should be kept confidential and authentic. If several cloud accesses are linked, and any of those are identified as, say, a create, then the adversary knows the government has added several classified files, which may, e.g., contain development plans. As a result, the adversary will know the government is planning to do something.

Enterprise: One key information of present business is the purchase of raw material. If the rival links several cloud accesses to the record of the same provider (e.g., quotation), he may change his business strategy because he knows his opponent's supplier.

Military: In modern battlefield, soldiers or military units can accept orders from tablet that connects to the cloud ${ }^{1}$. If the adversary detects a write on the cloud, he may conclude the strategy has changed, and thus get prepared to change his strategy accordingly.

Meta File: Frequency is a measure of importance. For example, one of the most frequently accessed data is probably the index (e.g., root folder). The server can deliberately delete such files in order to make the outsourced data unusable.

Stock Exchange: Consider the settings where a Stock Exchange department outsources his clients' profiles to the cloud. When the new comers pour in the Stock Exchange, the new money will drive the stock price up. In this sense, when the adversary sees the frequency of create rises, he can buy more shares in advance. When he sees a lot of delete operations, he needs to sell his shares.

The examples above indicate the necessity of protecting access pattern; this is the focus of this paper. We propose a security model to measure the necessity and construct the system accordingly. Moreover, we build an SSE system that is safer than any systems ever (with similar complexity).

\subsection{Related Works}

Searchable Symmetric Encryption was proposed by Song, Wagner, and Perrig in [23]. It enables a user to search over encrypted files (on the cloud). They also suggested re-encrypting the whole database periodically because of information leakage. Curtmola et al. [8] introduced semantically secure searchable encryption with search time sublin-

\footnotetext{
${ }^{1}$ Nowadays, military tends to use public cloud to reduce budget, otherwise, we consider the case the military cloud is hacked by the adversary.
}

ear to the number of files containing the queried keyword. They also introduced CKA2 (adaptive chosen keyword attack). Later, Kamara et al. [17] constructed an SSE scheme which is explicitly dynamic, but could leak more information. Recently, Cash et al [7]. facilitates counters and gave us a simple construction; Hahn and Kerschbaum [14] presented a more mature and consistent system based on the lazy learning idea; Naveed et al. [19] invented Blind Storage (BS) and gave a safer system.

BS was proposed as a primitive to support dynamic SSE by Naveed et al. In BS, each data item is stored as a collection of blocks that are kept in pseudorandom locations; the server sees only a super-set of the locations where the data item is stored, rather the exact set of locations. From the server's point of view, each data item is associated with a set of locations independent of the other items. (Indeed, the sets of locations for two files can overlap.) Using BS as a basic building block, Naveed et al. built a SSE system that achieves some security properties missing in classic SSE constructions.

However, since the cloud access of the 'virtual' access is deterministic, two cloud accesses are identical if the underlying 'virtual' accesses are the same. Thus, BS leaks access pattern. In fact, BS can hardly conceal access pattern at all.

In order to conceal access pattern, a trivial solution is to transfer the whole cloud storage if user wants to access some data items, but the high cost of this method is obviously unwanted.

Another approach is to use ORAM [13]. Initially invented to protect software, ORAM (Oblivious Random Access Machine) is a good tool that can totally conceal access pattern with logarithmic. Suppose there are $n$ items, an access to one item needs $\left((\log n)^{3}\right)$ accesses (each performing an access to one item), and there exists a background algorithm to shuffle the data in locations 'actually' accessed, in order to make any subsequent cloud accesses unlinkable. Although subsequent optimizations have been made these years $[9,11,21,25,26]$, the high cost (logarithmic to $n$ ) of it thwarts its adoption. As a result, recent works tends to make the key information oblivious [18] (and leave other information unprotected from leaking access patterns), or partition the information hierarchically [24].

\subsection{Our Contributions}

Standing between ORAM and BS, we propose AIS, which makes trade-offs between efficiency and security. AIS is the first construction that can 'partially' conceal access pattern to the best of our knowledge. Previous works either totally conceal access pattern but too costly, or hardly conceal access pattern.

We propose the first security definition $\mathcal{I N D}-\mathcal{C F} \mathcal{A}$ that can concisely capture the notion of 'partially' conceal access

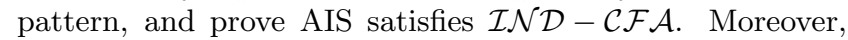
we propose a more powerful and meaningful definition $\mathcal{A P} \mathcal{I}$, and list it as a future direction to design efficient systems to satisfy this definition.

One application of AIS is SBA, an SSE system built on AIS. In SBA, the leakage function, which aims at measuring the information leakage, accumulates much less information than almost any previous constructions, except those built on ORAM. The key idea is using the lazy updating strategy to make each operation leak less information. The compu- 
tation and communication complexity of SBA, on the other hand, is only a multiple of the fastest state-of-the-art constructions (with more information leakage).

To summarize, the contributions are: First, the security model $\mathcal{I N D}-\mathcal{C} \mathcal{F} \mathcal{A}$ and $\mathcal{A P \mathcal { I }}$. Second, the construction of AIS. Third, the idea and construction of SBA.

The rest of this paper is organized as follows: In Section 2 , we give some preliminaries, and the system model of both AIS and SBA. Then we give a new security model for access pattern in Section 3. In Section 4, we give full construction of AIS. Finally, construct SBA on AIS in Section 5.

\section{PRELIMINARIES}

In this section, we will give overviews of systems settings of AIS and SBA respectively. We first itemize the notations we will use in Table 2 . Then we briefly describe the system settings and goals of both AIS and SBA.

\begin{tabular}{|c||c|}
\hline Symbol & Description \\
\hline$F=\{f\}$ & A file set. \\
\hline$f$ & An individual file. \\
\hline$i d_{f}$ & $\begin{array}{c}\text { The unique identifier associated } \\
\text { with a data item (file id). }\end{array}$ \\
\hline$|f|$ & Length of this file, including its identifier. \\
\hline content & $\begin{array}{c}\text { Plain (unencrypted or decrypted) data } \\
\text { transfered between user and client (file content). }\end{array}$ \\
\hline data $_{i}$ & $\begin{array}{c}\text { Encrypted fixed-sized data transfered between } \\
\text { client and server (encrypted file segment). }\end{array}$ \\
\hline index $_{i}$ & Location (of a fixed-sized block) on the cloud. \\
\hline$b=\$\{0,1\}$ & Challenger randomly choose a bit and assign it to $b$. \\
\hline$b \leftarrow \mathcal{A}$ & $b$ is the output of an algorithm $\mathcal{A}$. \\
\hline$Z_{n}$ & The set of $\{1,2, \ldots, n\}$. \\
\hline
\end{tabular}

Table 2: Notations used through this paper.

\subsection{System Model of AIS}

As shown in Figure 1, AIS is a client system that enables the user to access data on the cloud without leaking access patterns. It interacts with user and makes use of APIs provided by the cloud storage system like other client-side cloud software does. Now we elaborate what the figure conveys. AIS sits between the user and the cloud storage server, and converts between plain messages (communication between user and AIS) and encrypted messages (communication between AIS and server), thus to protect the user's data. We assume that the encrypted messages are available to the adversary.

We assume other information, such as the delay of each interaction, the length of the data, do not leak information about the communication. Actually, we can insert some noisy delay or dummy data, and this is beyond the scope of this paper.

The server may be controlled by the adversary, but since under the settings of cloud storage service, it is reasonable to just assume the server is honest but curious, ${ }^{2}$ which means

\footnotetext{
${ }^{2}$ If the server is malicious - this means the server will not follow our instructions at all - it is also easy to ensure security, just by inserting some authentication information pseudorandomly in the communication, however, this is not the focus of this paper.
}

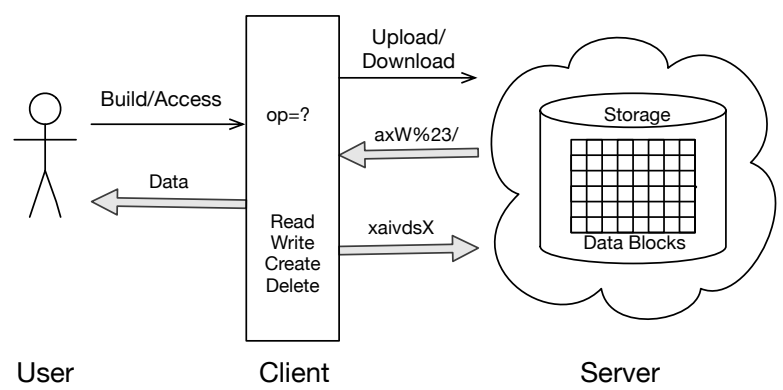

Figure 1: System model of AIS.

the server will follow the client's instructions but try to learn any information available, and the communication channel is safe (assume the channel uses TLS, and this is beyond the scope of this paper). In this sense, it is reasonable to regard the server as the (honest yet curious) adversary.

The server has a storage, whose size is specified by the client (and user). We assume the server storage is an array of fixed sized data blocks ${ }^{3}$. Each data block stores ciphertext encrypted by the client. The server provides two APIs to the client, namely, Upload and Download.

- $U p l o a d\left(\left\{\text { index }_{i}\right\}_{i \in Z_{n}},\left\{\text { data }_{i}\right\}_{i \in Z_{n}}\right)$ : The client specifies $n$ locations ${ }^{4}$, which are block indices in our system settings, and $n$ data items (encrypted). The size of each item equals to the block size of the cloud. The server, after receiving the indices and data items, stores the data items to the corresponding blocks in the cloud.

- Download $\left(\left\{\text { inde }_{i}\right\}_{i \in Z_{n}}\right)$ : The client specifies $n$ locations. The server, after receiving the locations, returns the corresponding data blocks in these locations.

There are two phases in the client, Build and Access. In Build stage, the user calls Build $\left(1^{\lambda}, F\right)$ only once, and the client generates private keys, prepares local data structures and gathers the initial data items. For simplicity, we regard such data items as files (each file has a unique id). But any kinds of data items, such as data blocks, or entries in a database, can be data items. Then client stores initial files to the server (after some transmission and encryption). In Access, user calls Access(op, $i d_{f}$, content), and client calls the corresponding underlying procedure according to the op parameter. There are four procedures as listed below. (When we say a file $i d_{f}$ exists on the cloud, we mean the encrypted format, rather than the plain format, has been stored on the cloud.)

- $\operatorname{Read}\left(i d_{f}\right)$ : User specifies an identifier $i d_{f}$. Client, after receiving $i d_{f}$, calculates locally and interacts with server. At last, client returns the content of this file (if exists) to user, in decrypted form. If $i d_{f}$ does not exist on the cloud, client returns empty.

- Write $\left(i d_{f}\right.$, content $)$ : User specifies a file identifier $i d_{f}$ and the new content content. After receiving the above

\footnotetext{
${ }^{3}$ If clouds don't support access to blocks, like Dropbox, we can create files of the same size to mimic the blocks.

${ }^{4}$ We can create files with sequential names, and use the file name as index
} 
parameters, client rewrites the file (specified by $i d_{f}$, if exists) with content. If $i d_{f}$ does not exist on the cloud, client does nothing.

- Create $\left(i d_{f}\right.$, content $)$ : User specifies a new file identifier $i d_{f}$ and content content. Client create a new file with name $i d_{f}$, and content content. If $i d_{f}$ is already stored on the cloud, client does nothing.

- Delete $\left(i d_{f}\right)$ : User specifies a file $i d_{f}$ to delete, and client deletes the file on cloud. If the file does not exist on the cloud, client does nothing.

In our settings, the client needs to maintain some local variables, so we assume that there is only one client in order to make the system simple. Some synchronization techniques will be needed if multiple clients are supported. This is a shortcoming of AIS.

To make our system model general enough, we assume conservatively that the cloud storage server provides only Upload and Download interfaces, although some cloud may support more complicated functionalities. Such assumptions may free the client from using the more expensive cloud processing servers, and make our system model general enough.

\subsection{System Model of SSE}

In the general settings of (Dynamic) SSE, data owner owns and outsources the data to the cloud. User (may be the data owner) uses some methods to search the keyword and get the corresponding data ${ }^{5}$. During the process, data owner may update the data, by interacting with the cloud, as shown in Figure 2.

In this paper, we adopt a simpler setting: We assume there is only one cloud, one data owner and one client. Moreover, the data owner is also the client. So we don't need to consider the communications between data owner and client. Moreover, the corresponding data received from the cloud is the file. Actually, the data has been encrypted by AIS. Note that supporting multiple users is feasible, but users have to synchronize in order to get the consistent state information. This is a shortcoming of our construction of SSE.

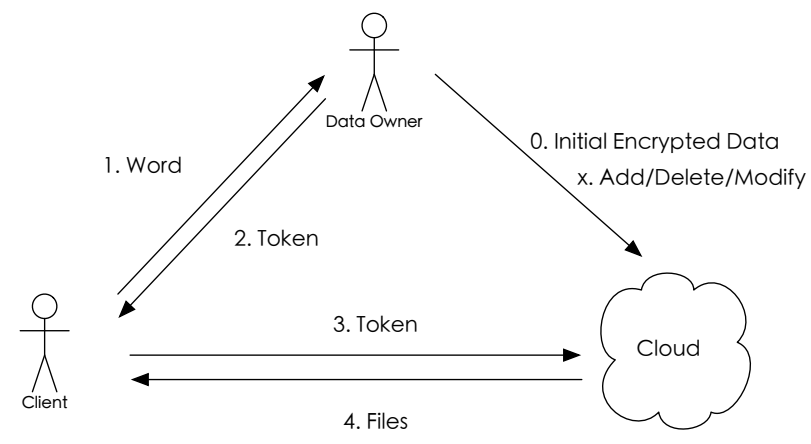

Figure 2: System model of SSE.

\footnotetext{
${ }^{5}$ In some schemes, two clouds are used. One cloud stores the dataset, while the other stores the index of the dataset. User queries one cloud for index and uses that to retrieve data (files) from the other.
}

\section{SECURITY MODEL}

In this section, we give a formal definition of $\mathcal{I N D}-\mathcal{C F} \mathcal{A}$ (indistinguishable under chosen file attack) security model. $\mathcal{I N D}-\mathcal{C} \mathcal{F} \mathcal{A}$ measures the ability to conceal access pattern.

First, we make several assumptions (abstractions) and provide oracles to make the security model clean. Note that these assumptions can be removed easily later. Our construction takes into account these assumptions.

- We do not conceal lengths of files during accessing and thus assume all files are of the same length. Bigger files can be split into several smaller files. The remaining segments of files can be padded.

- Operations such as creation and deletion can be modeled as write: We can create all files $U$ in the build phase. For a creation, we pick an empty file and write; for a deletion, we mark this file as empty (e.g., set a special tag or modify the file to all zeros). This works in reality because in the construction of AIS, we will write the whole space initially, and we will still write even we are deleting. Thus, we can assume that there're only read and write in our system, and all files are created for us to use after build phase. Moreover, we assume each file id is unique, which is required in common file systems.

- Finally we assume read and write are also indistinguishable. Thus, all operations are indistinguishable. We will see in the construction, and (informally) prove later, there's no difference in appearance between a read operation and a write operation. Thus focus only on whether we've succeeded in concealing the file accessed in the following model.

\begin{tabular}{|l|}
\hline Oracle Trigger \\
\hline $1: \quad$ content $\leftarrow \mathcal{A}$ \\
$2: \quad f=\$ U$ \\
$3: \quad U_{0}:=U_{0}-\{f\}, U_{1}:=U_{1} \cup\{f\}$ \\
$4: \quad$ AP $[t]:=($ content,$f)$ \\
$5: \quad$ Access $\left(\right.$ content,$\left.i d_{f}\right)$ \\
Oracle Indicate \\
\hline $1: \quad$ content $\leftarrow \mathcal{A}$ \\
$2: \quad f=\$ \mathcal{A}$ \\
$3: \quad U_{0}:=U_{0}-\{f\}, U_{1}:=U_{1} \cup\{f\}$ \\
$4: \quad$ AP $[t]:=($ content,$f)$ \\
$5: \quad$ Access $\left(\right.$ content,$\left.i d_{f}\right)$ \\
Oracle Open \\
\hline $1: \quad t \leftarrow \mathcal{A}$ \\
$2: \quad$ return AP $[t]$ \\
\hline
\end{tabular}

Note that Trigger is 'weak' than Indicate, but is useful in defining different levels of attackers.

Consider the following game, where $U$ is the universal file set, $F$ is a subset of $U, A P$ is the access pattern, content is something the attacker specifies to write, and Trigger, Indicate, Open are oracles given below: 


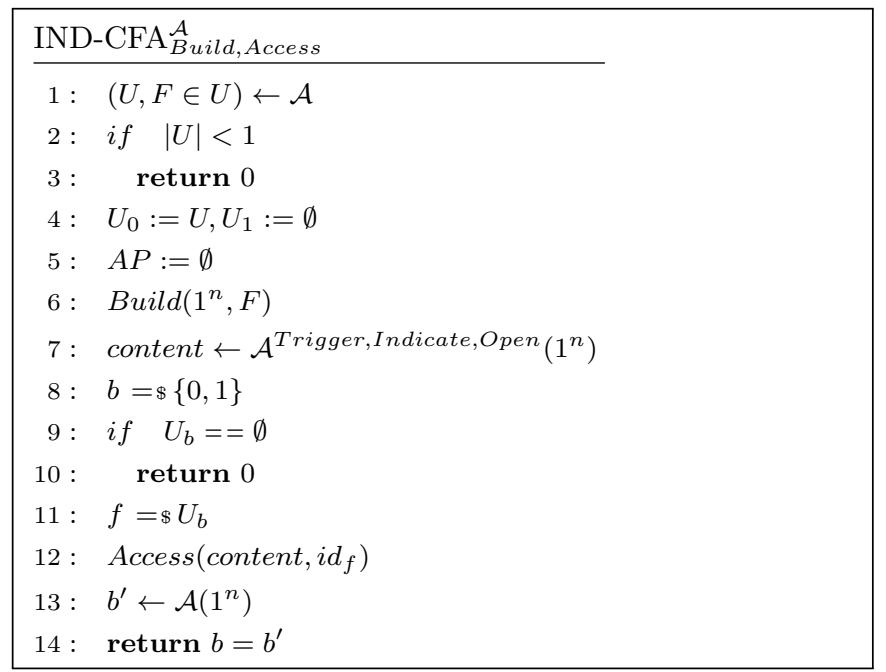

DEFINITION 1. (IND-CFA) We say a scheme $\mathcal{E}=($ Build, Access) is indistinguishable under adaptive chosen file attack if for all probabilistic polynomial-time algorithms $\mathcal{A}$, the advantage of $\mathcal{A}$ defined as

$$
\mid \operatorname{Pr}\left[1 \leftarrow \mathrm{IND}_{-\mathrm{CFA}}^{\mathcal{A}} \text { Build,Access }\right]-\frac{1}{2} \mid
$$

is negligible in $n$.

\section{Discussion.}

Under this attack model, the adversary will not know whether the current file being accessed by challenger was accessed before or not, since he cannot distinguish an access to an accessed file from an access to an unaccessed file. Note that, as is implicitly inferred in the game, re-encrypting the whole dataset after a period (in this game, before $U_{1}==U$ ) is useful for security, which is also wildly recognized by the community.

Given an access, $\mathcal{A}$ is allowed to have confidence (at most $50 \%$ ) about which file the client is accessing. However, $\mathcal{A}$ does not know for sure whether the client is accessing this file or another. This is useful though. For example, when a doctor is creating record for a patient, the adversary may guess the doctor is reading another known patient's record, and thus wrongly asserts that known patient has disease. This somehow 'false positive' thing is actually bad for the adversary.

Access Path Indistinguishable (API): To model stronger security guarantee, we propose API in Definition 2.

Definition 2. (API) We say a scheme $\mathcal{E}=($ Build, Access $)$ is indistinguishable under adaptive chosen file attack in PATH version if for all probabilistic polynomial-time algorithms $\mathcal{A}$, the advantage of $\mathcal{A}$ defined as

$$
\left|\operatorname{Pr}\left[1 \leftarrow \mathrm{IND}-\mathrm{CFA}-\mathrm{PAT} H_{\text {Build,Access }}^{\mathcal{A}}\right]-\frac{1}{2}\right|
$$

is negligible in $n$.

The game has similar settings as the previous one:

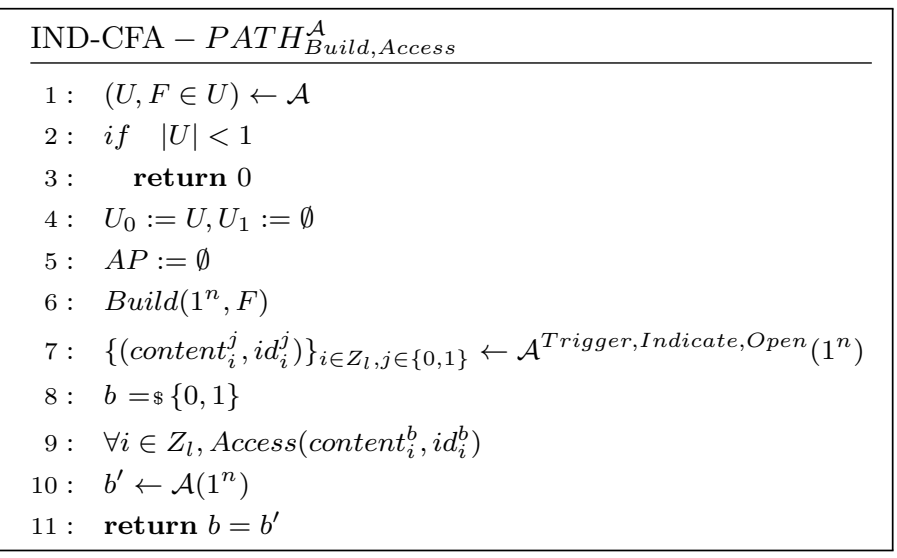

In this definition, we allow the adversary to specify two vectors (of length $l$ ) of parameters of Access procedure. The challenger randomly picks one to execute. Even with two paths $l$ accesses carefully prepared, the adversary still can't tell which path the challenger is executing after monitoring the cloud access pattern.

The relation between $\mathcal{A P \mathcal { I }}$ and ORAM is similar to that between witness indistinguishability (WI) and zero-knowledge $(\mathrm{ZK})$ in the foundations of cryptography [12]. We suggest the API definition is itself of independent value. We set the efficient constructions of API storage systems with constant overhead, compared to poly-logarithmic overhead of ORAM, as the goal of our future direction.

\section{ACCESS INDISTINGUISHABLE STOR- AGE}

In this section, we will go through the detailed construction of AIS. AIS is inspired by Blind Storage (BS) [19], but AIS can satisfy a much stronger security model.

In AIS, the server does not know:

- The exact operation of each access.

- Whether the file being accessed has been accessed before or not (after initialization).

- The exact number of files (though the server could get a bound based on the number of accesses, which is inevitable).

- The content of each file.

- The exact location of each file (though it might get a set of possible locations, which is inevitable).

- The number of initial files that are uploaded during initialization.

- The size of each initial file.

The last four are inherited from BS, but AIS can achieve the first three in addition. Moreover, in AIS, the size of each file during access phase can also be concealed, by splitting each file into parts of the same length (with padding) and access each part separately (each split is regarded as a file). However, sizes will be easily exposed if the access interval is long (boosted accesses will leak the size of the file, since they correspond to the same file, with high probability). We adopt this method here but ignore the security advantage. 
We will next give the intuition and reason for AIS, and the detailed construction. Analysis and security proofs are given in the end of this section.

\subsection{Overview and Intuition}

Now, we give a high-level idea of AIS: After we noticed that the deterministic BS only trivially conceals the information about initial files, we began to think about efficient storage system that can conceal access pattern even in access phase.

ORAM can achieve this, but we want to avoid its inefficiency. We noticed we can 'partially' conceal access pattern by obfuscating an access to a file that has been accessed before, with an access to a file that has not. To achieve this, we make sure each access overlaps half with a previous access, and the other is new. The idea is to pair an access to a random file that has been accessed, if the current intended file being accessed is new (create a new file, or access a file created in initial phase, but never accessed after initialization), and pair a dummy access to random locations if the intended file has been accessed after initialization.

Moreover, we add (pseudo)randomness by relocating the same file after each access, so that the next access may be different from previous ones (only a small fraction of overlap). To relocate the file, number of blocks accessed must be twice as before. Data in AIS is stored in the encrypted form, using a master secret key. To make sure each access to the same file looks different, a counter (works as an initial vector) is kept for each file to update the private key, which can be used (together with the file identifier) by a pseudorandom function to determine the indices (location) of the file.

Note that indices of different files may 'collide', so we need to generate $\beta$ (in our instance, $\beta=4$ ) times as many indices as needed to make sure there are enough free data blocks for a file to be stored (with probability high enough, proved in Theorem 1). When there is no enough space to store a file (happens with possibility negligible in security parameter), we can easily handle this by aborting current operation and storing again later, until success. To maintain simplicity of the system, we ignore such cases in construction. In total, AIS needs $2 \beta$ (constant) accesses for one data item. (Note that ORAM needs polylogrithmatic accesses, and BS needs $\beta$ accesses).

The efficiency we sacrifice for such security improvements is only a multiple of communication and computation. Specifically, we use roughly twice the network bandwidth and computational resource as BS, and roughly $2 \beta$ times for common storage systems (that does not provide security or privacy).

There are two phases of our algorithms: Build and Access. Build phase is simple. It just allocates local spaces and initializes local variables. Access phase is complicated. It deals with each kind of access operation separately. Each access consists of:

1. Calculate locations, and download corresponding data blocks from cloud.

2. Decrypt data blocks.

3. Write to these data blocks.

4. Encrypt data blocks.

5. Upload data blocks to to the same locations in cloud and refresh local variables.

\subsection{Construction}

Construction Architecture: AIS consists of three layers. The first layer (Figure 3) is provided to users. BuILD takes the security parameter and the initial file set, and initializes the cloud. ACCESs takes the operation op, file identifier $i d_{f}$, and file content content (if needed), and calls the second layer procedures (Figure 4). The second layer can handle the four kinds of operations separately and it calls both UPLOAD and DOWNLOAD for each part (split) of the file. UPLOAD and DOWNLOAD work as the last layer (Figure 5). Encryption and decryption are performed using the block index and the version of that block as keys. Moreover, DOWNLOAD redundantly downloads more data to satisfy the security model (as we can see later) and add more noise.

Detailed Construction: AIS is essentially a file IO component, so operations are performed in a way similar to file IO. There are several points to highlight: (The data structures and parameters used in the construction are elaborated in Table 3.)

In the build phase, BuILD basically takes the file set and write the files (locally), and then upload the whole file set.

In the access phase, we need to pair each access ( $\sigma$ from DownlOAD) with a dummy access $\left(\sigma^{\prime}\right)$. After the access, the real data may be written to its previous locations $\sigma$ or new locations $\sigma^{\prime}$. For new content (no previous location), however, an old access is paired (we should take care of the case when there is no old access as in line 9 of DownLOAD). In this case, the content is written to the new location for sure. Locations of the content are identified by location stored in $L$. For each split of a file, a seed is generated by $\mathrm{PRF}$, together with hash of the file identifier and its split. Then the seed is stretched by PRG as the final location indices. Dummy locations are generated by count by similar procedures. Moreover, count identifies location sets that are already seen. Here we permute the locations to improve security (line 15 in DownLOAD), but theoretically, this does not help.

Note the encryption and decryption schemes in line 17 of BUILD and line 4 of UPLOAD, and line 20 of DownLOAD requires $\mathrm{PRF}$, which is insufficient for block size. There are several ways to substitute this module: We can either use traditional encryption schemes taking $v$ and $i$ as initial vector, or repeat PRF (each time increment $v$ ).

Cloud size $C S$ should be specified explicitly in advance, and it should be at least $\beta$ times of whole data size. Users should re-initialize a new system when data size grows too big. (Note in the security model, we ignore this and always assume cloud size is big enough.) It is usually good practice to re-encrypt (re-initialize) the whole dataset again after some time of usage. AIS will warn users (line 11 of DOWNLOAD) after enough usage. Each block in the cloud contains $s$ bits of data, $4 R$ bits to identify the owner (which part of which file) of this block, and $R$ bits of version number (like initial vector). Only the version number is stored in plaintext.

\section{Extensions and Optimizations.}

Since a lot of redundant information is transferred and computed during each access, our AIS system can easily support PDP or POR (Provable Data Possession [3-5] or Proofs of Retrievability [10,16,20,22], respectively), by (pseudo)randomly inserting redundant information in each block. 


\begin{tabular}{|c|c|}
\hline Symbol & Description \\
\hline$C S$ & Cloud storage size. Storage is organized as an array of blocks. \\
\hline$N_{b}$ & Number of blocks in cloud. The blocks are indexed linearly. \\
\hline$s$ & Block size. $(s+R+4 R) \times N_{b}=C S . R=|v| \cdot$ \\
\hline$R$ & Length of a representation of an index. $R=\left\lceil\log N_{b}\right\rceil$. \\
\hline$\alpha$ & Split size in blocks. Each file is partitioned into parts of size $\alpha$ blocks. \\
\hline$\beta$ & Collision resistent full-domain hash function $:\{0,1\}^{*} \rightarrow\{0,1\}^{3 R}$. \\
\hline$H$ & Pseudorandom function $:\{0,1\}^{3 R+R} \rightarrow\{0,1\}^{4 R} \cdot$ \\
\hline$P R F$ & Pseudorandom generator $:\{0,1\}^{4 R} \rightarrow\{0,1\}^{R \alpha \beta}$. block. \\
\hline$P R G$ & Array of size $R$. Content of each slot is a number. \\
\hline$L^{\prime}$ & Array of size $R$. Content of each slot is an array of tuples, and each tuple is two numbers. \\
\hline$L$ & Set of files that have been accesses after initialization. \\
\hline$\Sigma$ &
\end{tabular}

Table 3: Data Structures and Parameters in AIS

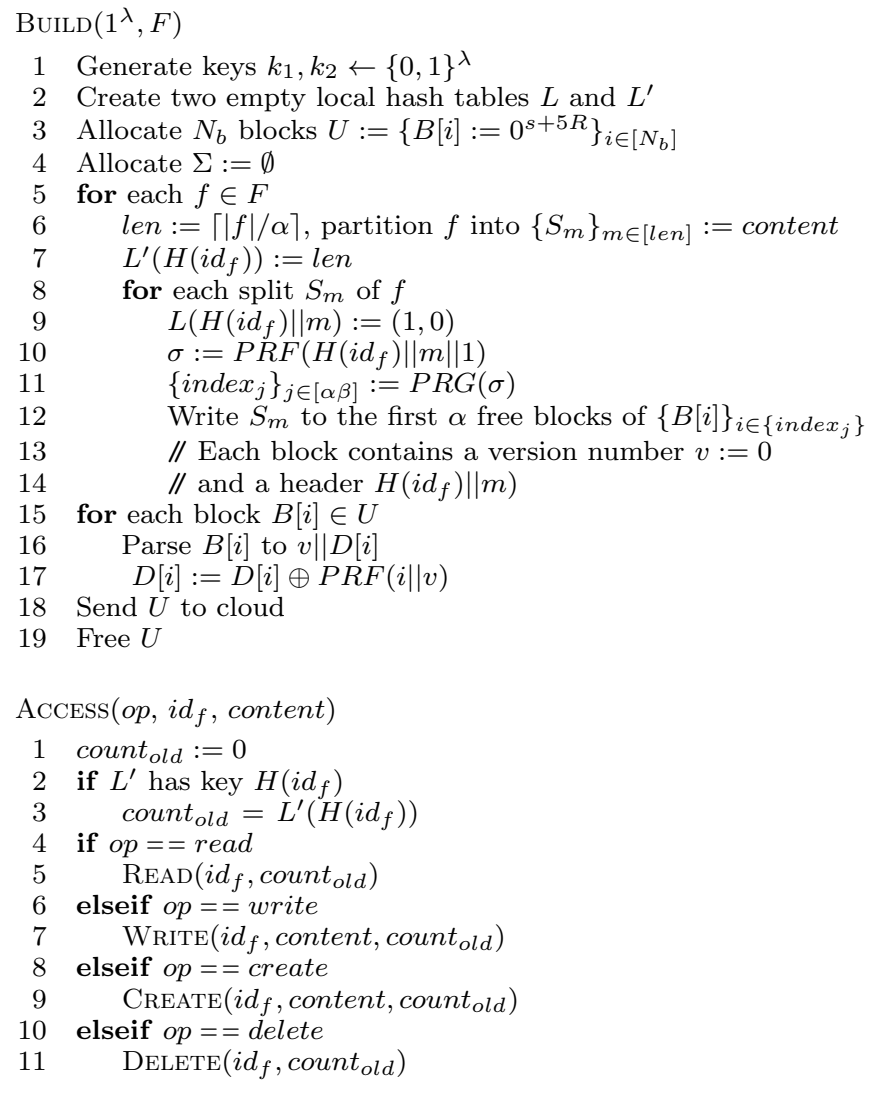

Figure 3: Two phases of AIS. These provide interfaces to end users.

One speedup technique is to allow the client to maintain an extra bitmap to indicate which blocks are empty and which are occupied, so that delete operation now won't require a redundant upload.

\subsection{Proofs and Analysis of AIS}

Since AIS adopts the idea of BS, as claimed in [19], the initial number of files and the length of initial files can be concealed. Moreover, since the four kinds of access operations look the same, they are indistinguishable from appear- ance. Further, AIS satisfies some stronger guarantee and we will prove each of them as follows. Some of them may refer the proof from BS [19].

THEOREM 1. The probability that a file can't be stored is negligible.

Proof. (Adopted from proof of Theorem 1 in BS [19].) Without loss of generalization, assume some proportion of the blocks are already allocated, and we define the ratio of allocated files to the size of cloud storage as $a$. Assume $a<\frac{1-1 / b}{2}$, where $b$ is a fixed integer larger than one (so this limits the usage of the cloud). Since we use pseudorandom generator to determine the locations of each file, we can assume the allocated blocks are randomly distributed on cloud. If the size of the current cloud accessing data if $b|f|$, then in expectation, $a b|f|$ of them may be occupied by other files. By Chernoff bound, the probability that $2 a b|f|$ of the accessing data is occupied by other files is less than $\left(\frac{e}{4}\right)^{a b|f|}$. When $|f|$ is super-logarithmic (linear) in the security parameter $\lambda$, the probability becomes negligible in $\lambda$. So the probability that there's $b|f|(1-2 a)>b|f|(1-(1-1 / b))=|f|$ spare space is close to 1 .

In actual implementation, $|f|=\alpha, b=\beta$. The upper bound of cloud usage is $\frac{1-1 / \beta}{2}$.

Property 1. AIS conceals the number of initial files and the length of each initial file.

Proof. (Inferred from BS [19].) After the build phase, all the blocks are uploaded in encrypted format. Thus, given the ciphertext, the adversary can't distinguish whether the plaintext is all zeros (this block is empty) or not (this block contains data). (Assuming the underlying encryption scheme, which is PRF here, is secure.) So the adversary can't tell the number of occupied blocks. Moreover, the adversary does not know the correlation between blocks, since all the version numbers are set to zero. In this sense, even if the adversary knows the number of allocated blocks, he will not know the number of initial files and the length of initial each initial file. $\square$

PROPERTY 2. The four kinds of access operations are indistinguishable.

Proof. This is trivial since the four different operations follow the same behavior (seen by cloud), and then we exclude the timing side channel. 

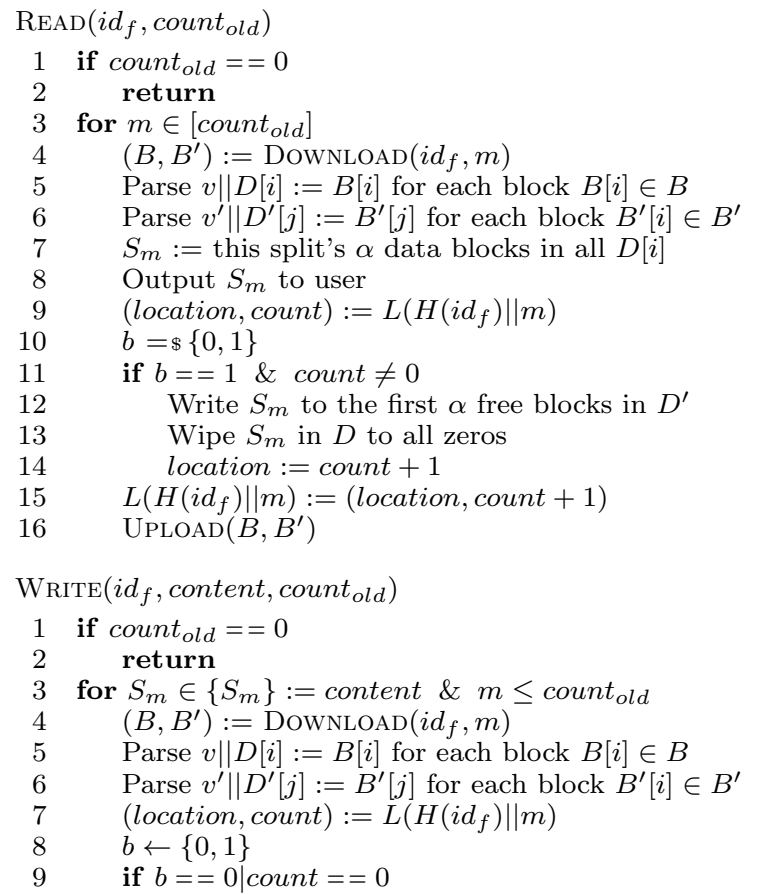
Write $S_{m}$ to $\alpha$ blocks belonging to it in $D$ else

Write $S_{m}$ to the first $\alpha$ free blocks to $D^{\prime}$ Wipe this split's $\alpha$ blocks in $D$ to all zeros location $:=$ count +1 $L\left(H\left(i d_{f}\right) \| m\right):=($ location, count +1$)$ $\operatorname{UPLOAD}\left(B, B^{\prime}\right)$

for $S_{m} \in\left\{S_{m}\right\} \& m>$ count $_{\text {old }}$ $L\left(H\left(i d_{f}\right) \| m\right):=(1,0)$

$\left(B, B^{\prime}\right):=$ DOWNLOAD $\left(i d_{f}, m\right)$

Parse $v \| D[i]:=B[i]$ for each block $B[i] \in B$ Write $S_{m}$ to $\alpha$ blocks belonging to it in $D$ $\operatorname{UpLOAD}\left(B, B^{\prime}\right)$

for $m:=\lceil\mid$ content $\mid /(\alpha s)\rceil+1$ to count $_{\text {old }}$ $\left(B, B^{\prime}\right):=\operatorname{DownLOAD}\left(i d_{f}, m\right)$ Parse $v \| D[i]:=B[i]$ for each block $B[i] \in B$ Wipe this split's $\alpha$ blocks in $D$ to all zeros Erase the entry $L\left(H\left(i d_{f}\right) \| m\right)$ $\operatorname{UpLOAD}\left(B, B^{\prime}\right)$

$L^{\prime}\left(H\left(i d_{f}\right)\right):=\lceil\mid$ content $\mid /(\alpha s)\rceil$

Create $\left(i d_{f}\right.$, content, count $\left._{\text {old }}\right)$

if count $_{\text {old }} \neq 0$ return

for $S_{m} \in\left\{S_{m}\right\}:=$ content

$L\left(H\left(i d_{f}\right) \| m\right):=(1,0)$

$\left(B, B^{\prime}\right):=\operatorname{DownLOAD}\left(i d_{f}, m\right)$

Parse $v \| D[i]:=B[i]$ for each block $B[i] \in B$

Parse $v^{\prime}|| D^{\prime}[j]:=B^{\prime}[j]$ for each block $B^{\prime}[i] \in B^{\prime}$

Write $S_{m}$ to the first $\alpha$ free blocks in $D$

$L\left(H\left(i d_{f}\right) \| m\right):=(1,1)$

$\operatorname{UPLOAD}\left(B, B^{\prime}\right)$

$L^{\prime}\left(H\left(i d_{f}\right)\right):=\lceil\mid$ content $\mid /(\alpha s)\rceil$

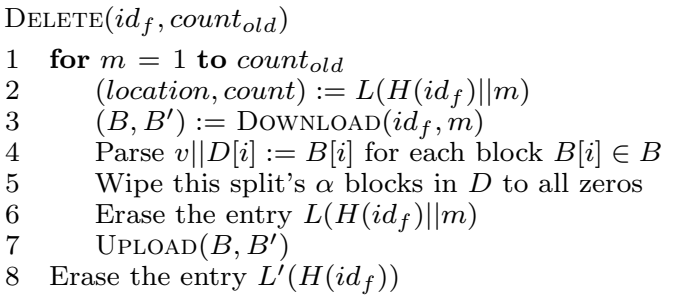

Figure 4: Four kinds of underlying operations.

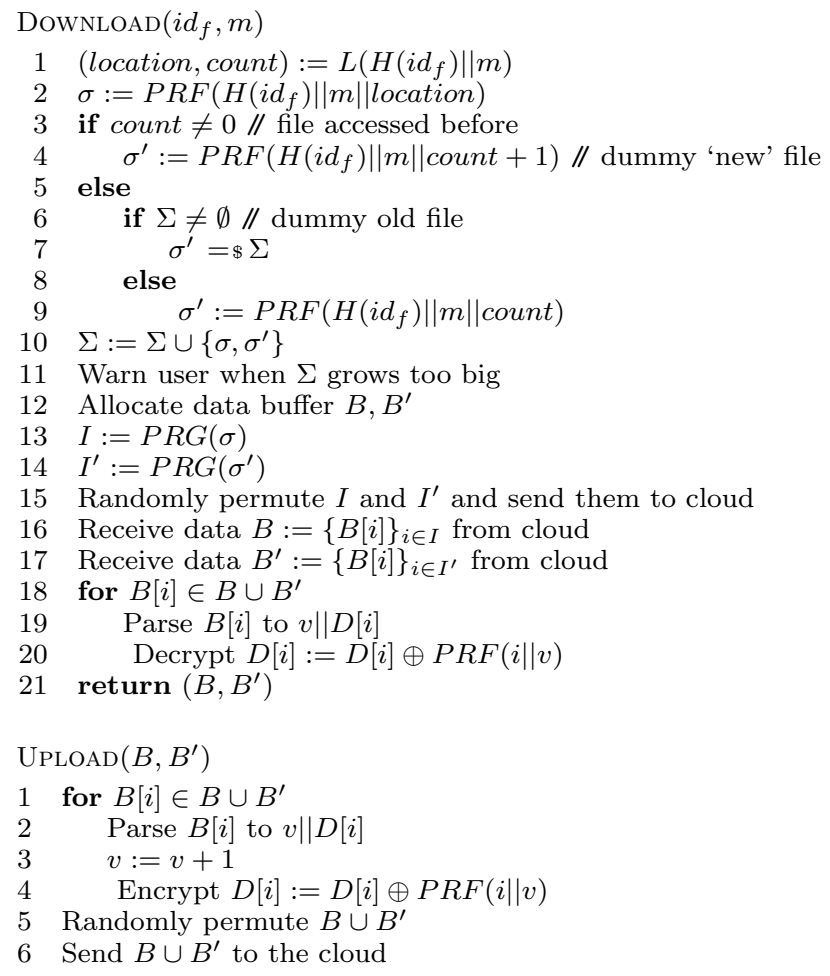

Figure 5: Lower level APIs works as infrastructure.

Property 3. Files that have been accessed will not leak information about files that have not been accessed.

Proof. The determination of each file's locations is independent, but may overlap. So revealing the location of one file will not exclude the other files from residing in (some of) the same blocks. Moreover, such revealings will not leak information of files' locations either, since the locations are determined independently, by a pesudorandom generator using file identifier and a counter. So, revealing the locations of one file (one access) will not lead to revealing any information about other files not accessed before.

ThEOREM 2. AIS is indistinguishable under chosen-file attack (Definition 1).

Proof. (Sketch) For simplicity, we informally give the intuition of the proof: In the construction of AIS, each time we access a split (file in $\mathcal{I N D}-\mathcal{C F} \mathcal{A}$ ), we are actually accessing two splits. So each 'virtual' access is an 'actual' access to the 'real' locations plus an 'actual' access to the 'dummy' locations. The 'real' locations are calculated by the pseudorandom generator, taking the file identifier and a counter (location) as the seed. If the access is new (create, or access the initial file for the first time), the 'dummy' ones correspond to a random previous access; otherwise, if the file has been accessed, the 'dummy' locations are calculated taking a new counter as the seed. In this sense, except for the first access after the build phase (which we exclude in the definition), half of each access has been seen before, and half not. It is unclear to the adversary whether the current access is new or old, therefore AIS satisfies $\mathcal{I N D}-\mathcal{C F} \mathcal{A}$. 


\section{Complexity of AIS.}

Communication bandwidth is only a constant ( $4 \beta-)$ blowup of information needed. Specifically, both UPLOAD and DownLOWD are needed for each operation, and each accesses $2 \beta$ times of blocks as needed.

Computation needed for each access is a constant number of hash, PRF, PRG, and array lookups, which are fast enough on AES enabled hardwares.

Local storage needed is linear in data size, but is much smaller. Specifically, local storage is bounded by number of splits. When $C S=4 G B, s=4 K B, \beta=4$, cloud usage is bounded by $1 G B$ (at most around $1.5 G B$ ), so there are $256 K$ splits. Approximately, the overall local storage should not exceed several megabytes. Note that this can scale to larger clouds. E.g., when $C S=32 T B, s=4 M B, \beta=4$, tens of megabytes is enough. Note this is a trade-off between local storage and padding size (block size).

\section{SSE BUILT ON AIS}

In this section we construct a searchable encryption scheme, SBA, from AIS. The high-level idea of SBA is to leverage the security provided by AIS, as defined in Definition 1, to reduce information leakage and provide more features. Another key idea of SBA is the lazy updating strategy which could minimize the information leakage. As a result, SBA is safer than previous schemes except those built on ORAM, but is more efficient than those built on ORAM. We first give the construction, then we define the leakage function and measure the information leakage.

\subsection{Construction of SBA}

Before giving the algorithms of SBA, we informally give an overview of the system. SBA uses AIS as modules, and we assume information transferred between AIS and SBA is in plain text, since both AIS and SSE run on the client side. (If SBA and AIS resides in different entities, we need to use secure channels.) Moreover, for the simplicity of the algorithms, we do not contain fault-tolerance in our construction.

Construction Overview: For simplicity of elaboration, we adopt two AIS, one (AIS-I) for storing the indices (identifier is keyword, and content is file identifiers), and the other (AIS-F) for storing files. One can also treat index of each keyword as a common file in AIS, by adding specific prefix or suffix to distinguish index files from common files. For simplicity, we do not need to concern cloud size of AIS used here. But SBA can be easily modified to handle these concerns.

There are two phases of SBA. In the first phase, the data owner (user) outsources his data by BuILD procedure. In the second phase, users (for simplicity, we assume there is only one user, and this is the same as data owner) query keywords and get result documents (documents containing the keyword). Currently, similar to other SSE schemes, SBA only supports simple query (single keyword). Data owner (user) can also update the dataset during the second phase.

Key Idea: We adopt the lazy updating methods. Specifically, when adding a file, we do not update the whole index immediately, instead, we gradually update index for each word when searching for that word. Modification and deleting files are similar. By adopting these methods, our system leaks minimal information, while maintains high efficiency.
Detailed Construction: To support the lazy updating strategy, a hash table $D$ is used to map each keyword to a timestamp of last query. This time can be used to find the files updated after last query. Thus, update operation does not need to concern about index update. Instead, during query time, the queried index will be refreshed itself. To achieve this, we maintain a (doubly linked) list $T$ that stores timestamps and the corresponding updated file identifier (the list is ordered by timestamps). We also maintain an auxiliary hash table (not shown in the algorithm) to map file identifier to the element in the list $T$, to accelerate searching. ( $T$ and the auxiliary hash table can be substituted by other structures such as Red-Black tree.)

The resultant algorithm is quite simple: For each query, we just need to find the time of its last query, and find the files updated after that time, check these files, and update the index. One optional storage optimization, as shown in the algorithm is, when the file does not contain any new

$\operatorname{Build}(F)$

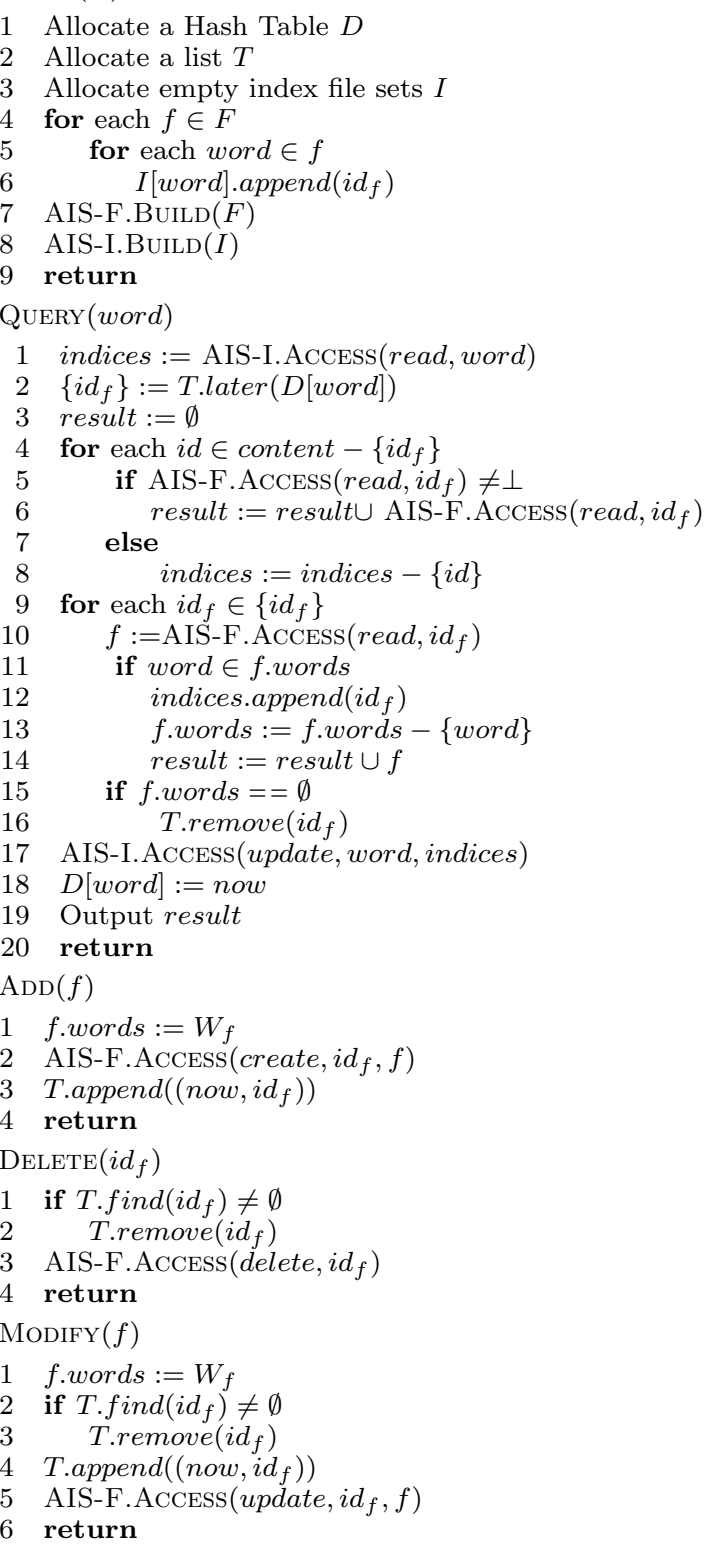

Figure 6: User level interfaces of SBA. 
documents, it can be removed from $T$. For the update operations, we just need to update the file, and do not need to worry about index update. The only thing to concern is update $T$ (remove old item if exists, and append a new one).

Specifically, for the deleted files, read as before and check. If this file cannot be found in AIS, (check in line 5 of QUERY), it is deleted, and we update the index (line 8 of QUERY). For the newly added files, we check if it contains the searched keyword (line 11 of QUERY), and update the index if it is true. We store an auxiliary information f.words to each file, in order to avoid unnecessary searches, if all distinct words of this file is searched, it is removed from $T$ (line 16 of QUERY). Note that this is a module of optimization, and can be changed or discarded.

For the added or modified files, we first get the time it was last searched, and get the set of files added or modified between that time and now (line 2 of QUERY), and check each of them.

Observation and Intuition: The intuition of this design is that: it is impossible to hide an update, but the content of each updated file is sensitive. Moreover, it is hard to hide the length of a file, so we adopted the lazy updating strategy mentioned above. As a result, the information leakage is nearly optimal, as we can see below.

\subsection{Leakage Functions and Security Definition}

Leakage function concisely measures the information leaked by the SSE scheme. We define leakage functions of SBA and compare them with some other major constructions, in Table 4 .

$A(w)$ and $D(w)$ are defined as the files (both $I D_{f}$ and $|f|$ ) added/modified or deleted between last query (or initial) and this query of $w$. Note that this leakage is unavoidable, and other constructions (BS and SUISE) leak more information in the add/modify or delete operations. SUISE can support file modification by a deletion followed by an addition, and the leakage function is defined as the combination of both, while ours can support file modification explicitly.

We follow the mainstream real/ideal simulation paradigm [8] and parameterize the scheme by our leakage functions.

DEFINITION 3. Consider the following experiments of a stateful PPT adversary $\mathcal{A}$, a stateful simulator $\mathcal{S}$ and leakage functions as defined in Table 4, define games $\operatorname{Real}_{A}(\lambda)$ and $\operatorname{Ideal}_{A, S}(\lambda)$ as follows:

- Real ${ }_{A}^{S B A}(\lambda)$ : The adversary $\mathcal{A}\left(1^{\lambda}\right)$ chooses a file set $F$, calls $S B A$.Build $(F)$, and adaptively chooses polynomial valid requests $q$. Each $q$ can be a search, an add, a delete, or a modify request, and the parameters needed are chosen by $\mathcal{A}$. $S B A$ will handle the requests one by one, and for each request, $\mathcal{A}$ can see the transcript (behavior and communication) of the cloud (and can adaptively choose the next request). Moreover, for a search request, $\mathcal{A}$ can get $I$, the set of file identifiers (encrypted), as the search result. Finally, $\mathcal{A}$ outputs a bit $b$.

- Ideal ${ }_{A, S}^{S B A}(\lambda)$ : The adversary $\mathcal{A}\left(1^{\lambda}\right)$ chooses a file set $F$. The simulator $\mathcal{S}$ gets $L_{B u i l d}$ and initializes its internal state. $\mathcal{A}$ then chooses polynomial valid requests $q$ adaptively. Each $q$ can be a search, an add, a delete, or a modify request, and the parameters needed are chosen by $\mathcal{A}$. For each request, $\mathcal{S}$ will get $L_{\text {search }}, L_{\text {add }}$,
$L_{\text {delete }}, L_{\text {modify }}$ accordingly. Then $\mathcal{S}$ interacts with the cloud and outputs the transcript (communication and behavior) of the cloud to $\mathcal{A}$, which based on this information adaptively choose the next request. Moreover, for a search request, $\mathcal{A}$ can get I, the set of file identifiers (without literal meanings), as the search result. Finally, $\mathcal{A}$ outputs a bit $b$.

$S B A$ is secure L-secure ${ }^{6}$ against adaptive chosen keyword attacks if for all adversaries $\mathcal{A}$ there exists an algorithm $\mathcal{S}$ such that the possibility

$$
\left|\operatorname{Pr}\left[\operatorname{Real}_{A}(\lambda)=1\right]-\operatorname{Pr}\left[\operatorname{Ideal}_{A, S}(\lambda)=1\right]\right|
$$

is negligible in $\lambda$.

\subsection{Proofs and Analysis of SBA}

Property 4. SBA conceals whether the current access corresponds to an addition, a modification or a deletion.

Proof. Because of Property 2 of AIS, together with the fact that the three kinds of file update functions, namely, addition, modification, and deletion behave identical (access a file), it is trivial that the adversary cannot tell apart the three operations. Note that we do not consider timing or other kinds of side-channel attacks.

PROPERTY 5. SBA conceals whether the current file being accessed has been accessed before.

Proof. According to Theorem 2 of AIS, together with the fact the three kinds of operations behave similarly (Property 4), and do not touch indices, if we are accessing a file (result of query), the adversary cannot tell whether this file was accessed before.

Property 6. SBA conceals whether a keyword has been queried before or not, if there's no new files added after the build phase, in the setting where we do not need to transfer file identifiers containing the queried keyword.

Proof. When we do not need to read, or reveal the identifiers of the files containing the keyword, according to Theorem 2 , it is infeasible to tell whether the index file we are accessing has been accessed before or not.

Discussion: It is also possible to eliminate the restrictions of the property above, but the adversary's confidence may become very high. For example, when a file is added, each query afterwards should read this file exactly once and compare whether this keyword is contained in this file (until all keywords contained in this file is queried). In this sense, after a file is added, (assuming no query in between) first query of a keyword should always read that file, but second query should not. Moreover, when we need to transfer the identifiers or read the files, this is also hard since identifiers are identical. For two consecutive queries of the same keyword, both of them will access the same set of files (assuming no file update). For the two accesses of each file, half of the locations will overlap. In both cases, the adversary can tell with high confidence the second queried keyword has been queried.

${ }^{6} L$ is the set of all leakage functions, i.e., $L=$ $\left\{L_{\text {build }}, L_{\text {search }}, L_{\text {add }}, L_{\text {modify }}, L_{\text {delete }}\right\}$ 


\begin{tabular}{|c||c|c|c|}
\hline Operation & BS $[19]$ & SUISE $[14]$ & SBA \\
\hline \hline$L_{\text {Build }}(F)$ & $N$ & $\left\{\left\{\left(I D_{f},\left|W_{f}\right|\right): f \in F\right\}\right\}$ & $N$ \\
\hline$L_{\text {Search }}(w)$ & $\left\{I D_{w},\left\{\left(I D_{f},|f|\right): w \in W_{f}\right\}\right\}$ & $\left\{I D_{w},\left\{\left(I D_{f},|f|\right): w \in W_{f}\right\}\right\}$ & $\left\{I D_{w},\left\{\left(I D_{f},|f|\right): w \in W_{f}\right\}\right.$ \\
\hline$L_{\text {Add }}(f)$ & $\left\{I D_{f},\left\{I D_{w}: w \in W_{f}\right\}\right\}$ & $\left\{I D_{f},|f|,\left|W_{f}\right|,\left\{I D_{w}: w \in S, w \in W_{f}\right\}\right\}$ & $\left\{I D_{f},|f|\right\}$ \\
\hline$L_{\text {Delete }}\left(I D_{f}\right)$ & $\left\{I D_{f},\left\{I D_{w}: w \in W_{f}\right\}\right\}$ & $\left\{I D_{f},|f|,\left|W_{f}\right|,\left\{I D_{w}: w \in S, w \in W_{f}\right\}\right\}$ & $\left\{I D_{f},|f|\right\}$ \\
\hline$L_{\text {Modify }}\left(I D_{f}, f^{\prime}\right)$ & - & - & $\left\{I D_{f},|f|,\left|f^{\prime}\right|\right\}$ \\
\hline
\end{tabular}

Table 4: Leakage function comparison. Unless specified otherwise, $f \in F$, the total file set, which is changed during addition or deletion. $I D_{f}$ can be regarded as the encrypted token of file identifier $i d_{f}$, and $I D_{w}$ can be regarded as the encrypted token of $w$.

THEOREM 3. SBA is secure under chosen keyword attack as described in Definition 3.

Proof. We define a simulator $\mathcal{S}$ who, on the one hand, receives requests and the corresponding leaked information, while on the other hand, using two AIS systems interacts with the cloud, and outputs transcripts to $\mathcal{A}$. Although $\mathcal{S}$ does not know the exact data $(\mathcal{S}$ only knows the information leaked by leakage functions), $\mathcal{A}$ still cannot distinguish $\mathcal{S}$ from the real SBA system. Now we define the behavior of $\mathcal{S}$ on receiving each of the leakage functions.

- Lbuild: $\mathcal{S}$ receives $N$, the size of the cloud storage, and then initializes two AIS systems with all empty files: AIS - I.Build $(\emptyset), A I S-F . B u i l d(\emptyset) . \mathcal{S}$ then initializes local variables $S_{w}:=\emptyset$ as the set of keyword-index pairs, $T_{w}:=\emptyset$ as the set of all keyword-time pairs, $S_{F}:=\emptyset$ as the set of all added files, and $A_{F}:=\emptyset$ to be the set of all added file-time pairs

- $L_{\text {search }}$ :

1. $\mathcal{S}$ receives the identifier of the keyword being searched $I D_{w}$, and the (original) index (only identifier) of files contain this keyword, $I, A(w), D(w)$.

2. If $I D_{w} \in S_{w}$, call $A I S-I$.Access $\left(\right.$ read, $\left.I D_{w}\right)$, otherwise, call AIS-I.Access (create, $I D_{w}, 1^{|I|+|D(w)|}$ Where $|I|$ here defines the number of tuples in $I$.

3. $\forall\left(I D_{f},|f|\right) \in I+D(w)$, if $I D_{f} \in S_{F}$, call $A I S-$ F.Access $\left(\right.$ read, $\left.I D_{f}\right)$; otherwise, call AIS - F.Access (create, $\left.I D_{f}, 0^{|f|}\right), S_{F}:=S_{F} \cup$ $I D_{f}$.

4. $\forall\left(I D_{f},|f|\right) \in A^{\prime}(w)$, if $I D_{f} \in S_{F}$, call $A I S-F . A c c e s s\left(\right.$ read, $\left.I D_{f}\right)$; otherwise, call $A I S-$ F.Access (create, $\left.I D_{f}, 0^{|f|}\right), S_{F}:=S_{F} \cup I D_{f} . A^{\prime}(w)$ is files added between last access (or initial) to now, by enumerating files in $A_{F}$ after $t$, where $t:=T_{w}\left(I D_{w}\right)$.

5. Call AIS - I.Access(update, $\left.I D_{w}, 0^{|I|}\right)$, and output $I$ to $\mathcal{A}$.

6. Add $\left(I D_{w}, t\right)$ to $T_{w}$, or update to $\left(I D_{w}, t\right)$ if $\left(I D_{w}, t^{\prime}\right)$ already exists in $T_{w}$.

- $L_{a d d}$ : Get an identifier of the file to be added, $I D_{f}$, and the length of that file, $|f|$. If $I D_{f} \in S_{F}$, call AIS - F.Access (update, $I D_{f}, 0^{|f|}$ ); otherwise, call AIS - F.Access (create, $\left.I D_{f}, 0^{|f|}\right), S_{F}:=S_{F} \cup I D_{f}$. Add $\left(I D_{f}, t\right)$ to $A_{F}$, or update to $\left(I D_{f}, t\right)$ if $\left(I D_{f}, t^{\prime}\right)$ already exists in $A_{F}$, where $t$ is the current time.

- $L_{\text {modify }}$ : Get an identifier of the file to be added, $I D_{f}$, the length of the old and new files, $|f|$ and $\left|f^{\prime}\right|$, respectively. If $I D_{f} \in S_{F}$, call $A I S-F$.Access(update, $I D_{f}, 0^{|f|}$; otherwise, call AIS-F.Access(create, $\left.I D_{f}, 0^{|f|}\right), S_{F}:=$ $S_{F} \cup I D_{f}$. Add $\left(I D_{f}, t\right)$ to $A_{F}$, or update to $\left(I D_{f}, t\right)$ if $\left(I D_{f}, t^{\prime}\right)$ already exists in $A_{F}$, where $t$ is the current time.

- $L_{\text {delete }}:$ Get an identifier of the file to be deleted, $I D_{f}$ and the length of that file, $|f|$. If $I D_{f} \in S_{F}$, call $A I S-F$.Access (delete, $\left.I D_{f}\right)$, and remove $I D_{f}$ from $S_{F}$; otherwise ( $f$ is one of the initial files), call $A I S-$ F.Access (create, $\left.I D_{f}, 0^{|f|}\right), S_{F}:=S_{F} \cup I D_{f}$. $\mathcal{S}$ finally removes $\left(I D_{f}, t^{\prime}\right)$ from $A_{F}$ if $\left(I D_{f}, t^{\prime}\right)$ exists in $A_{F}$; where $t$ is the current time.

The distribution of the transcript without knowing the secrets (files, searched keywords) should be the same as the transcript knowing the secrets, according to the definition of AIS.

\section{Complexity of SBA.}

As with AIS, communication bandwidth is the most concerning issue since it is always the bottleneck of the system. Fortunately, the overall communication complexity is nearly optimal. Specifically, each update operation (ADD, DELETE, -MODIFY) only needs one call of AIS. For the QUERY procedure, we can analyze it reversely: Each updated file will be accessed by every keyword once, rather than those contained by the updated file. Suppose there are $m$ keywords in universe, and $m_{f}$ distinct words in in updated file $f$. Now in worst case, there are $m-m_{f}$ unnecessary access (compare and find word $\notin f$ ) in our design. Instead, if we update the corresponding indices (but will leak more information, namely, keywords contained in $f$ ), $m_{f}$ accesses are enough. Note that this is necessary to achieve better privacy: now the adversary does not know which words (and number of distinct words) are contained in this document. However, since SBA and AIS both run at client side, communication overhead is not a concern.

Computation complexity is low in SBA. To find the set of files updated between two queries takes $O(|F|)$ time, and other operations take constant time. It is also possible to use other data structures to achieve better computation complexity. Since SBA relies on AIS, the complexity of SBA is bounded by that of AIS.

Local storage is not much. We need to store each keyword and updated files (in a hash table, and a list and a hash table, respectively). Again, the space complexity here is bounded by AIS. One thing to explore is to run AIS on non-colluding cloud (or trusted third party). By doing that, computation and storage are not longer concerns, but communication becomes the bottleneck. 


\section{CONCLUSIONS AND FUTURE WORK}

In this paper, we present AIS and SBA. We give security models, constructions, proofs, analysis and comparisons for both AIS ans SBA. To the best of our knowledge, both AIS and SBA are better than state-of-the-art constructions, to some extent. Moreover, we proposed API security model (defined in Definition 2) of independent value.

There are several future directions: First, developing constantoverhead path-AIS that resists attacks of any polynomial number of steps and satisfies API. Another direction of future work is to make the client stateless. Currently, the client needs to keep information about all the files on cloud. Even AIS and SBA can scale well, when multiple users want to access the cloud together, synchronization is required, which introduces lots of traffic. To transfer such state information to the cloud should be meaningful and interesting.

\section{REFERENCES}

[1] Dropbox. https://www.dropbox.com.

[2] Google drive. https://www.google.com/drive/.

[3] G. Ateniese, R. Burns, R. Curtmola, J. Herring, L. Kissner, Z. Peterson, and D. Song. Provable data possession at untrusted stores. In Proceedings of the 14th ACM SIGSAC Conference on Computer and Communications Security, pages 598-609. ACM, 2007.

[4] G. Ateniese, R. Di Pietro, L. V. Mancini, and G. Tsudik. Scalable and efficient provable data possession. In Proceedings of the 4th international conference on Security and privacy in communication netowrks, page 9. ACM, 2008.

[5] K. D. Bowers, A. Juels, and A. Oprea. Hail: a high-availability and integrity layer for cloud storage. In Proceedings of the 16th ACM SIGSAC Conference on Computer and Communications Security, pages 187-198. ACM, 2009.

[6] D. Cash, P. Grubbs, J. Perry, and T. Ristenpart. Leakage-abuse attacks against searchable encryption. In Proceedings of the 22nd ACM SIGSAC Conference on Computer and Communications Security, pages 668-679. ACM, 2015.

[7] D. Cash, J. Jaeger, S. Jarecki, C. S. Jutla, H. Krawczyk, M. Rosu, and M. Steiner. Dynamic searchable encryption in very-large databases: Data structures and implementation. In 21st Annual Network and Distributed System Security Symposium, 2014.

[8] R. Curtmola, J. Garay, S. Kamara, and R. Ostrovsky. Searchable symmetric encryption: improved definitions and efficient constructions. In Proceedings of the 13th ACM SIGSAC Conference on Computer and Communications Security, pages 79-88. ACM, 2006.

[9] J. Dautrich, E. Stefanov, and E. Shi. Burst oram: Minimizing oram response times for bursty access patterns. In USENIX Security, volume 14, 2014.

[10] Y. Dodis, S. Vadhan, and D. Wichs. Proofs of retrievability via hardness amplification. In Theory of cryptography, pages 109-127. Springer, 2009.

[11] C. Gentry, K. A. Goldman, S. Halevi, C. Julta, M. Raykova, and D. Wichs. Optimizing oram and using it efficiently for secure computation. In Privacy Enhancing Technologies, pages 1-18. Springer, 2013.
[12] O. Goldreich. Foundations of Cryptography - Basic Techniques. Cambridge University Press, 2001.

[13] O. Goldreich and R. Ostrovsky. Software protection and simulation on oblivious rams. Journal of the ACM, 43(3):431-473, 1996.

[14] F. Hahn and F. Kerschbaum. Searchable encryption with secure and efficient updates. In Proceedings of the 21st ACM SIGSAC Conference on Computer and Communications Security, pages 310-320. ACM, 2014.

[15] M. S. Islam, M. Kuzu, and M. Kantarcioglu. Access pattern disclosure on searchable encryption: Ramification, attack and mitigation. In 19th Annual Network and Distributed System Security Symposium, 2012.

[16] A. Juels and B. S. Kaliski Jr. Pors: Proofs of retrievability for large files. In Proceedings of the 14th ACM SIGSAC Conference on Computer and Communications Security, pages 584-597. ACM, 2007.

[17] S. Kamara, C. Papamanthou, and T. Roeder. Dynamic searchable symmetric encryption. In Proceedings of the 19th ACM SIGSAC Conference on Computer and Communications Security, pages 965-976. ACM, 2012.

[18] C. Liu, A. Harris, M. Maas, M. Hicks, M. Tiwari, and E. Shi. Ghostrider: A hardware-software system for memory trace oblivious computation. In Proceedings of the International Conference on Architectural Support for Programming Languages and Operating Systems, 2015.

[19] M. Naveed, M. Prabhakaran, C. Gunter, et al. Dynamic searchable encryption via blind storage. In 2014 IEEE Symposium on Security and Privacy, pages 639-654, 2014.

[20] H. Shacham and B. Waters. Compact proofs of retrievability. In Advances in Cryptology-ASIACRYPT, pages 90-107. Springer, 2008.

[21] E. Shi, T.-H. H. Chan, E. Stefanov, and M. Li. Oblivious ram with $o((\log n) 3)$ worst-case cost. In Advances in Cryptology-ASIACRYPT, pages 197-214. Springer, 2011.

[22] E. Shi, E. Stefanov, and C. Papamanthou. Practical dynamic proofs of retrievability. In Proceedings of the 20th ACM SIGSAC Conference on Computer and Communications Security, pages 325-336. ACM, 2013.

[23] D. X. Song, D. Wagner, and A. Perrig. Practical techniques for searches on encrypted data. In 2000 IEEE Symposium on Security and Privacy, pages 44-55, 2000.

[24] E. Stefanov, C. Papamanthou, and E. Shi. Practical dynamic searchable encryption with small leakage. IACR Cryptology ePrint Archive, 2013:832, 2013.

[25] E. Stefanov and E. Shi. Oblivistore: High performance oblivious cloud storage. In 2013 IEEE Symposium on Security and Privacy, pages 253-267, 2013.

[26] E. Stefanov, M. Van Dijk, E. Shi, C. Fletcher, L. Ren, $\mathrm{X}$. Yu, and S. Devadas. Path oram: An extremely simple oblivious ram protocol. In Proceedings of the 20th ACM SIGSAC Conference on Computer and Communications Security, pages 299-310. ACM, 2013. 